\title{
SÍNTESE DE NANOMATERIAIS DE CARBONO A PARTIR DO RESÍDUO DE MILHO (DDGS)
}

\author{
Joner Oliveira Alves* \\ Centro de Pesquisa, Aperam South America, Praça $1^{\circ}$ de Maio, 35180-018 Timóteo - MG, Brasil \\ Chuanwei Zhuo e Yiannis Angelo Levendis \\ Department of Mechanical and Industrial Engineering, College of Engineering, Northeastern University. 360 Huntington Ave., \\ Boston, MA, 02115, EUA \\ Jorge Alberto Soares Tenório \\ Departamento de Engenharia Metalúrgica e de Materiais, Escola Politécnica, Universidade de São Paulo. Av. Prof. Mello Moraes, \\ 2463, 05508-030 São Paulo - SP, Brasil
}

Recebido em 13/11/11; aceito em 23/4/12; publicado na web em 20/7/2012

\begin{abstract}
SYNTHESIS OF CARBON NANOMATERIALS FROM CORN WASTE (DDGS). The world's largest ethanol producer (USA) uses corn as feedstock. DDGS (distillers dried grains with solubles) is the main waste generated from this process (around $32 \mathrm{million} \mathrm{t} /$ year). DDGS samples were pyrolyzed at $1000{ }^{\circ} \mathrm{C}$ in a furnace with controlled atmosphere. The effluent was channeled to a second furnace, in which catalyst substrates were placed. Chromatographic analysis was used to evaluate the gaseous effluents, showing that the catalyst reduced hydrocarbon emissions. The solid products formed were analyzed by SEM and TEM. Graphitic structures and carbon nanofibers, $50 \mu \mathrm{m}$ in length and with diameters of 80-200 $\mathrm{nm}$, were formed.
\end{abstract}

Keywords: nanomaterials; DDGS; pyrolysis.

\section{INTRODUÇÃO}

O interesse pela energia da biomassa tem aumentado consideravelmente devido à necessidade de fontes de energia renováveis e limpas. O etanol proveniente da biomassa possui potencial para substituir grandes quantidades de combustíveis derivados do petróleo. A redução dos gases do efeito estufa tem sido amplamente discutida devido ao aquecimento global; o emprego do etanol em substituição aos combustíveis fósseis é considerado uma das principais medidas a serem adotadas, uma vez que os combustíveis fósseis são responsáveis por $73 \%$ da produção de $\mathrm{CO}_{2} \cdot{ }^{1}$ As propriedades do etanol, como o teor de oxigênio (cerca de $35 \%$ do total em massa), possibilitam uma combustão com melhor desempenho dos motores e menores índices de poluição, mesmo quando misturado à gasolina. ${ }^{2}$

De acordo com a Global Renewable Fuels Alliance (GRFA), a produção mundial de etanol em 2010 foi de aproximadamente $8,6 \times 10^{10} \mathrm{~L}$, representando um aumento da produção de $16,2 \% \mathrm{em}$ relação ao ano anterior. ${ }^{3}$ A indústria do etanol é tradicionalmente liderada por EUA e Brasil, responsáveis por cerca de $90 \%$ da produção mundial. A China apresentou um crescimento acelerado nos últimos anos, embora ainda apresente volume de produção distante dos líderes mundiais. A principal matéria-prima para o etanol empregada no Brasil é a cana-de-açúcar, enquanto que nos EUA predomina a aplicação do grão de milho. ${ }^{4}$

A produção de etanol a partir do grão de milho apresenta crescimento consolidado nos últimos anos, em 2006 cerca de $1,9 \times 10^{10} \mathrm{~L}$ foram produzidos nos EUA, enquanto que, em 2009, foi registrada uma expansão para $3,8 \times 10^{10} \mathrm{~L}^{5}$ As atuais políticas de incentivos fiscais por parte do governo norte-americano tendem a aumentar ainda mais a competitividade deste setor, criando uma maior expectativa de produção para os próximos anos. ${ }^{6}$ Maior produtor mundial de etanol ( $46 \%$ do total), os EUA possuem o milho como matéria-prima base para $98 \%$ das indústrias produtoras deste combustível. A produção de etanol norte-americana se concentra na região chamada de Cinturão

*e-mail: joner.alves@ aperam.com do Milho, com destaque para os estados de Iowa e Illinois. ${ }^{5}$

O grão de milho é convertido em etanol, basicamente, por dois processos: moagem úmida ou moagem seca. Na moagem úmida, a semente de milho é fracionada em componentes primários (amido, gérmen e fibra), gerando coprodutos variados. No processo de moagem a seco existem seis etapas majoritárias: moagem, cozimento, liquefação, sacarificação, fermentação e separação. Os produtos finais da moagem a seco incluem álcool combustível, dióxido de carbono e os grãos secos de destilaria com solúveis. Devido à menor necessidade de investimentos e ao maior rendimento de etanol, as plantas de moagem seca são responsáveis por mais de $70 \%$ da produção de etanol baseado no milho. ${ }^{7}$

Assim como no etanol de cana, durante a fermentação, a glicose é transformada em etanol pela ação da levedura Saccharomyces cerevisiae. O líquido produzido é destilado e segue para um conjunto de centrífugas, no qual é separada a parte fina (que pode ser recirculada no processo) e a parte restante segue para evaporadores, nos quais é produzido o xarope (com cerca de $50 \%$ de umidade). $\mathrm{O}$ xarope é misturado a sólidos retirados na centrífuga e secado dando origem ao DDGS - distillers dried grains with solubles (grãos secos de destilaria com solúveis). ${ }^{8}$

O emprego do milho como matéria-prima para a produção de etanol apresenta um rendimento industrial de $460 \mathrm{~L}$ de etanol anidro e $380 \mathrm{~kg}$ de DDGS por cada tonelada de milho seco inserida no sistema. ${ }^{9}$ Atualmente, o DDGS é usado como ração animal devido ao elevado teor de proteínas. Entretanto, devido ao também elevado teor de fibras, esta aplicação se encontra limitada para a dieta de ruminantes, uma vez que o excesso pode causar problemas de saúde. O DDGS deve ser estocado em galpões adequados para que não perca as propriedades nutricionais, fato que aumenta o custo com este material. ${ }^{10}$

A geração de DDGS tem aumentado em função do aumento na produção de etanol, sendo que somente em 2009 as indústrias norte-americanas produziram cerca de 3,2 x $10^{7} \mathrm{t}$ deste material. ${ }^{11}$ Estudos apontam que a indústria do etanol tende a continuar crescendo, gerando aumento na produção do DDGS. Segundo O'Brien ${ }^{12}$, em cerca de 5 anos a produção de DDGS nos EUA poderá chegar a cerca de 
Tabela 1. Análise química do DDGS empregado no trabalho (em \% p.)

\begin{tabular}{ccccccccccccc}
\hline $\mathrm{C}$ & $\mathrm{O}$ & $\mathrm{Si}$ & $\mathrm{H}$ & $\mathrm{Fe}$ & $\mathrm{K}$ & $\mathrm{Ca}$ & $\mathrm{S}$ & $\mathrm{Cl}$ & $\mathrm{P}$ & $\mathrm{N}$ & $\mathrm{Outros}$ \\
\hline 48,2 & 17,8 & 0,3 & 7,5 & 0,5 & 7,6 & 0,6 & 6,7 & 1,5 & 3,2 & 3,7 & 2,4 \\
\hline
\end{tabular}

4,5 x $10^{7}$ t. Portanto, é desejável encontrar novas destinações para o DDGS, visando agregar valor a este produto e, consequentemente, aumentar o custo/benefício da cadeia produtiva do etanol a partir do milho.

O reaproveitamento do DDGS como combustível estabelece fontes de energia e vapor, uma vez que este material possui um teor energético de $27 \mathrm{MJ} / \mathrm{kg}$, o que excede o conteúdo energético dos carvões lignosos (10-20 MJ/kg) e se aproxima dos carvões betuminosos $(30 \mathrm{MJ} / \mathrm{kg})$, atualmente empregados no setor industrial. ${ }^{13}$ Neste trabalho, foi investigado um sistema de catálise dos gases gerados durante a queima do DDGS, visando a redução das emissões gasosas e a produção de nanomateriais.

A nanotecnologia tem despertado o interesse de diversas áreas devido às enormes possibilidades de aplicações. Os nanomateriais possuem graus estruturais na ordem de nanômetros $\left(10^{-9} \mathrm{~m}\right){ }^{14}$ Diversos tipos de nanomateriais, como os nanotubos de carbono, nanopartículas metálicas, nanopartículas de óxidos de metais de transição, nanoemulsões e diferentes tipos de nanocompósitos, deixaram de ser alvo apenas dos laboratórios acadêmicos e podem facilmente ser encontrados em produtos comercializados atualmente. Em 2010, produtos que empregam nanotecnologia movimentaram cerca de US\$11 trilhões mundialmente; deste montante, US $\$ 340$ bilhões corresponderam apenas aos nanomateriais como, por exemplo, catalisadores de automóveis, materiais para gravação magnética e componentes para protetores solares..$^{15,16}$

\section{PARTE EXPERIMENTAL}

A matéria-prima empregada foi o DDGS, fornecido por uma indústria de etanol do estado de Iowa, EUA. O material foi recebido na forma de grãos com diâmetros de $\sim 10 \mathrm{~mm}$. A Tabela 1 apresenta a análise química do DDGS utilizado.

Uma navícula de alumina foi utilizada para inserir $4 \mathrm{~g}$ do material no sistema. $\mathrm{O}$ aparato empregado consistiu em dois fornos tubulares, em escala laboratorial, ligados por um tubo de quartzo. Nos experimentos foi empregado o processo de pirólise, no qual o material é decomposto por tratamento térmico com a ausência de gases oxigenados, para tanto foi mantida uma atmosfera controlada pelo fluxo de $\mathrm{N}_{2}$ (vazão de $3 \mathrm{~L} / \mathrm{min}$ ).

As amostras foram inseridas no forno primário, no qual ocorreu o processo de pirólise do material a uma temperatura de $1000^{\circ} \mathrm{C}$. Os produtos da pirólise foram submetidos a um filtro de carbeto de silício (SiC) com estrutura do tipo colmeia, que retém 97\% das partículas submícron. O efluente gasoso passante do filtro foi canalizado para o forno secundário, pré-aquecido a $1000{ }^{\circ} \mathrm{C}$. A Figura 1 ilustra o aparato empregado no trabalho.

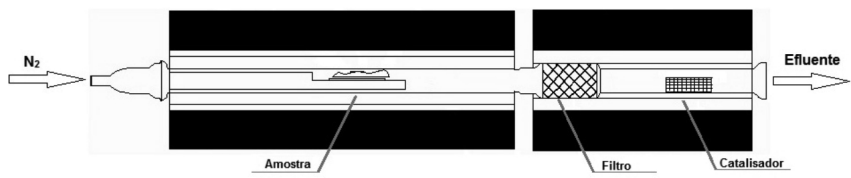

Figura 1. Desenho esquemático do aparato utilizado nos experimentos

No forno secundário, previamente, foram inseridas telas metálicas de forma paralela à direção do fluxo de gases para atuarem como catalisador, seguindo a teoria da catálise de transferência de fase (atuação de um agente transferidor para reação entre substâncias em diferentes fases). As telas foram produzidas com o aço inoxidável AISI 304, uma liga constituída de $\mathrm{Cr}(18-20,0 \%)$, $\mathrm{Ni}(8-10,5 \%), \mathrm{Mn}(\leq 2,0 \%)$, Si $(\leq 1,0 \%), N(\leq 0,1 \%), C(\leq 0,08 \%), \mathrm{P}(\leq 0,05 \%), \mathrm{S}(\leq 0,03 \%)$ e $\mathrm{Fe}$ (balanço). ${ }^{17} \mathrm{O}$ material foi utilizado sem pré-tratamentos, apenas com limpeza por acetona. Peças de 300 x $100 \mathrm{~mm}$ foram modeladas com formato cilíndrico com diâmetros de $40 \mathrm{~mm}$.

As caracterizações consistiram em duas frentes, uma análise dos efluentes gasosos gerados e um estudo dos materiais sintetizados. Para a análise dos gases, amostras com volume de $0,5 \mathrm{~L} / \mathrm{min}$ foram coletadas com o uso de seringas acionadas por um injetor eletrônico posicionado na saída do forno secundário. As amostras coletadas foram analisadas em um aparelho de cromatografia gasosa modelo HP-6890, com detectores de ionização de chama e condutividade térmica (GC-FID/TCD) e equipado com duas colunas paralelas. Foram analisados os gases da pirólise do DDGS com e sem o uso de tela metálica, visando uma avaliação da influência do sistema catalisador através da comparação dos resultados.

Para a caracterização dos materiais sólidos produzidos foram retiradas amostras de diferentes regiões da tela metálica, que foram analisadas em um microscópio eletrônico de varredura (MEV), modelo Hitachi 4800, e em dois tipos de microscópio eletrônico de transmissão (MET), um de baixa resolução (modelo JEOL 1010 tensão de aceleração de $70 \mathrm{kV}$ ) e um de alta resolução (modelo JEOL 2010 - tensão de aceleração de 200 kV).

\section{RESULTADOS E DISCUSSÃO}

\section{Avaliação das emissões gasosas e da influência do sistema catalisador}

Os hidrocarbonetos leves (LHCs) podem ser definidos como compostos orgânicos com baixo peso molecular e que consistem inteiramente de hidrogênio e carbono. Os principais hidrocarbonetos leves gasosos provenientes da queima do DDGS consistiram em metano, etileno, acetileno e benzeno, sendo que traços de outros compostos também foram detectados: tolueno, etano, propano, propileno, etilacetileno e etilbenzeno. A Tabela 2 exibe as emissões registradas com e sem a utilização das telas metálicas. O emprego do sistema catalisador reduziu as emissões dos hidrocarbonetos e aumentou o volume produzido de $\mathrm{H}_{2}$. Tal fato indica uma retenção de LHCs no sistema de catálise, ou seja, os gases gerados durante a pirólise do DDGS sofreram reação química quando em contato com a tela metálica.

Tabela 2. Emissões dos LHCs majoritários e $\mathrm{H}_{2}$ gerados durante os experimentos com e sem a tela catalisadora (em $\mathrm{mg} / \mathrm{g})$

\begin{tabular}{cccc}
\hline & Sem a tela & Com a tela & Diferença \\
\hline Metano & 75 & 55 & 21 \\
Etileno & 13 & 10 & 2 \\
Acetileno & 28 & 25 & 3 \\
Benzeno & 40 & 35 & 5 \\
LHCs (total) & 156 & 125 & 31 \\
\hline $\mathrm{H}_{2}$ & 3 & 6 & 3 \\
\hline
\end{tabular}

O processo de síntese empregado é baseado na deposição química de vapor (CVD), a qual consiste em reações de desidrogenação térmica (Equação 1) através do uso de um catalisador composto por 
metais de transição empregado para "quebrar" os hidrocarbonetos gasosos em $\mathrm{C}_{\text {(sólido) }}$ e $\mathrm{H}_{2}{ }^{18}$

$$
\mathrm{C}_{\mathrm{x}} \mathrm{H}_{\mathrm{y}} \rightarrow \mathrm{xC}+1 / 2 \mathrm{y} \mathrm{H}_{2}
$$

Baker et al. ${ }^{19}$ estudaram o crescimento de fibras de carbono através da decomposição catalítica de fontes de hidrocarbonetos nas superfícies ativas de metais de transição. Durante este processo, os hidrocarbonetos são decompostos em carbono e hidrogênio, seguido de uma difusão do carbono na superfície do metal, resultando em uma solução saturada. A supersaturação da solução leva à precipitação de partículas sólidas de carbono na superfície do metal. Outras pesquisas ${ }^{20-23}$ mostraram que os hidrocarbonetos podem ser utilizados como fonte de carbono na produção de nanomateriais empregando a metodologia CVD.

$\mathrm{O}$ metano $\left(\mathrm{CH}_{4}\right)$ foi o LHC que apresentou a maior concentração nos experimentos; este gás é o hidrocarboneto termodinamicamente mais estável, sendo o primeiro a sofrer decomposição no processo de pirólise em altas temperaturas. ${ }^{11}$ Kong et al.${ }^{20}$ investigaram a utilização do metano como combustível para produzir nanomateriais via CVD com temperatura de $1000{ }^{\circ} \mathrm{C}$, tendo sido produzidos nanotubos de carbono de paredes simples. Com base no que foi discutido, é possível afirmar que a recuperação dos hidrocarbonetos gasosos gerados durante a pirólise do DDGS como fonte de carbono para a síntese de nanomateriais carbonosos é uma tecnologia viável.

\section{Análise microestrutural dos materiais formados}

As caracterizações com o emprego da microscopia eletrônica de varredura objetivaram uma avaliação preliminar sobre a formação ou não dos nanomateriais. Portanto, para cada amostra com ganho de massa considerável após o experimento foi realizada uma análise por MEV. O MET foi empregado para uma maior definição das estruturas dos materiais formados. Os resultados da análise microestrutural estão dispostos nas Figuras 2 (obtidas via MEV) e 3 (obtidas via MET).

A Figura $2 \mathrm{a}$ exibe a tela catalisadora coberta pelos materiais sintetizados. Como pode ser observado nas Figuras $2 \mathrm{~b}$ e 2c, os materiais possuem formas retorcidas e irregulares, com uma superfície aparentemente escamosa. As ramificações dos nanomateriais mostraram-se impregnadas na superfície da tela metálica de um modo não uniforme, confirmando a teoria proposta anteriormente de que os nanomateriais foram formados nas partículas sólidas de carbono precipitadas na superfície do catalisador.

Analisando as imagens obtidas com o MET, Figura 3, é possível constatar a formação de dois tipos de materiais: nanofibras de carbono e grafita. Foram formadas nanofibras de carbono com comprimentos da ordem de $50 \mu \mathrm{m}$ e diâmetros entre 80 e 200 nm, as Figuras 3a e 3b são as que melhor demonstram este fato. Estruturas semelhantes foram relatadas por Vander Wal et al..$^{24}$ durante a síntese de materiais, através de hidrocarbonetos gasosos como matéria-prima e com o emprego do aço inoxidável como catalisador. As nanofibras de carbono são
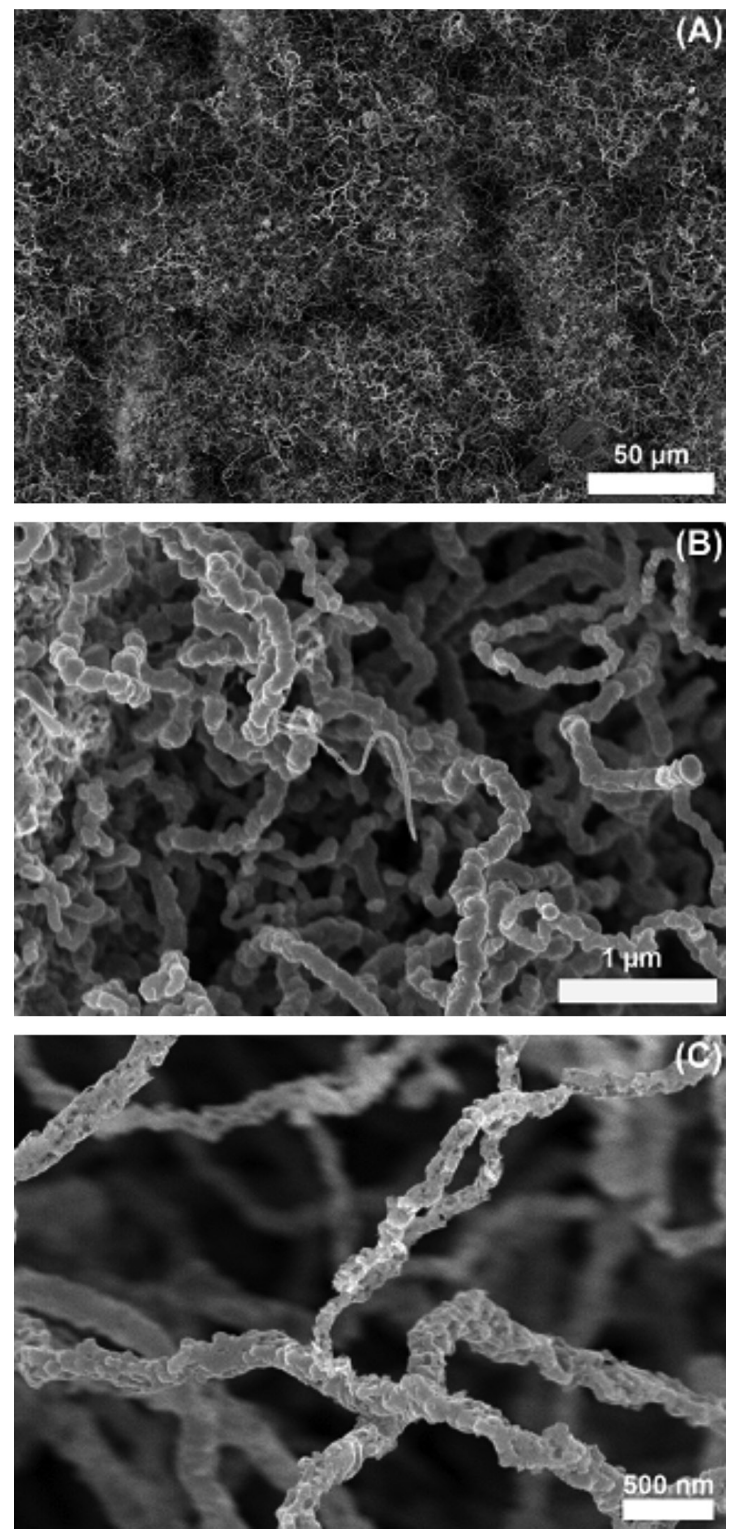

Figura 2. Imagens obtidas por MEV dos materiais sintetizados a partir dos gases de pirólise do DDGS
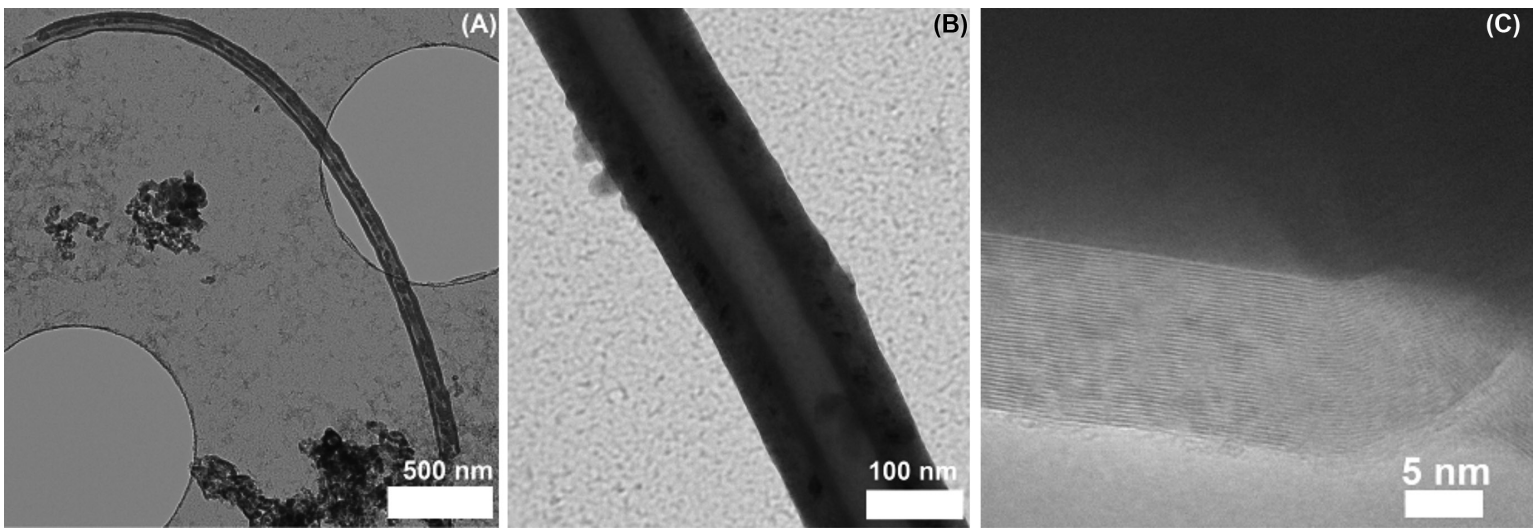

Figura 3. Imagens obtidas por MET dos materiais sintetizados a partir dos gases de pirólise do DDGS 
materiais compostos por placas de grafite empilhadas ao longo de um eixo com formato de cone. ${ }^{25,26}$ Através de uma análise da Figura 3c (obtida no microscópio de transmissão com alta resolução) é possível observar o enfileiramento das placas de grafite. Outra estrutura formada apresentou paredes corrugadas, com curvaturas acentuadas e superfícies irregulares, como observado nas Figuras 2c e 3a. Esta estrutura possui a forma da grafita, uma das formas alotrópicas do carbono, assim como o diamante, fulerenos, fibras e nanotubos. A grafita pode ser encontrada na forma mineral ou obtida sinteticamente através de fontes de carbono. ${ }^{26}$

\section{CONCLUSÕES}

Os principais hidrocarbonetos leves gasosos provenientes da pirólise do resíduo de milho, DDGS, consistiram em metano, etileno, acetileno e benzeno. $\mathrm{O}$ emprego da tela de aço inoxidável como catalisador ocasionou a decomposição dos hidrocarbonetos em carbono e hidrogênio. O carbono liberado nesta reação foi recuperado na forma de partículas sólidas, nas quais foram formadas estruturas grafíticas e nanofibras de carbono com comprimentos da ordem de $50 \mu \mathrm{m}$ e diâmetros de 80-200 nm. Portanto, os gases da queima do DDGS foram empregados com sucesso para a produção de nanomateriais de carbono, estabelecendo um destino rentável para o resíduo e uma fonte de matéria-prima de baixo custo para os nanomateriais.

\section{AGRADECIMENTOS}

Ao CNPq pelo financiamento do período do DSc. Joner Alves na Northeastern University (EUA), à CAPES pelo suporte ao projeto "Novos processos e produtos de nanotecnologia aplicados ao meio ambiente" (edital 04/CII-2008 - Rede Nanobiotec-Brasil) e à empresa Global Ethanol (Lakota, IA - EUA) pelo fornecimento do DDGS.

\section{REFERÊNCIAS}

1. Hoogwijk, M.; Faaij, A.; Vries, B.; Turkenburg, W.; Biomass. Bioenerg. 2009, 33, 26

2. BNDES, CGEE; Bioetanol de cana-de-açúcar: energia para o desenvolvimento sustentável, $1^{\text {a }}$ ed., Ed. Senac Rio: Rio de Janeiro, 2008.
3. Alves, J. O.; Zhuo, C.; Levendis, Y. A.; Tenório, J. A. S.; Appl. Catal., $B$ 2011, 106, 433.

4. Alves, J. O. Em Nanotecnologia para o Mercosul: Edição 2010 do Prêmio Mercosul de Ciência e Tecnologia; UNESCO, MBC, RECyT/ MERCOSUL, CNPq, eds.; UNESCO: Brasília, 2010.

5. Piñeiro, G.; Jobbágy, E. G.; Baker, J.; Murray, B. C.; Jackson, R. B.; Ecol. Appl. 2009, 19, 277.

6. Sadhukhana, J.; Mustafaa, M. A.; Misailidisb, N.; Mateos-Salvadora, F.; Dub, C.; Campbell, G. M.; Chem. Eng. Sci. 2008, 63, 503.

7. Kim, S.; Dale, B. E.; Biomass. Bioenerg. 2009, 28, 475.

8. Xu, W.; Reddy, N.; Yang, Y.; Carbohydr. Polym. 2009, 76, 521.

9. Wyman, C. E; Handbook on bioethanol: production and utilization, Applied Energy Technology Series, Taylor \& Francis: Washington, 1996.

10. Wu, F.; Munkvold, G. P.; J. Agric. Food Chem. 2008, 56, 3900.

11. Zhuo, C.; Alves, J. O.; Tenório, J. A. S.; Levendis, Y. A.; Ind. Eng. Chem. Res. 2012, 51, 2922.

12. http://www.agmrc.org/renewable_energy/agmrc-renewable-energynewsletter, acessada em Setembro 2010.

13. Wang, L.; Hanna, M. A.; Weller, C. L.; Jones, D. D.; Energy Conv. Manag. 2009, 50, 1704.

14. Zarbin, A. J. G.; Quim. Nova 2007, 30, 1484

15. Pitkethly, M. J.; Nano Today 2003, 36, 36.

16. Baughman, R. H.; Zakhidov, A. A.; Heer, W. A.; Science 2002, 297, 787.

17. ASTM; ASTM E2016 - 06 Standard specification for industrial woven wire cloth, ASTM International: West Conshohocken, 2006.

18. See, C. H.; Harris, A. T.; Ind. Eng. Chem. Res. 2007, 46, 997.

19. Baker, R. T. K.; Harris, P. S.; Thomas, R. B.; Waite, R. J.; J. Catal. 1973, 30,86 .

20. Kong, J.; Cassell, A. M.; Dai, H.; Chem. Phys. Lett. 1998, 292, 567.

21. Tibbetts, G. G.; Appl. Phys. Lett. 1983, 42, 666.

22. Zhao, N.; He, C.; Jiang, Z.; Li, J.; Li, Y.; Mater. Lett. 2006, 60, 159.

23. Alves, J. O.; Zhuo, C.; Levendis, Y. A.; Tenório, J. A. S.; Mater. Res. 2011, 14, 499.

24. Vander Wal, R. L.; Hall, L. J; Berger, G. M.; J. Phys. Chem. B 2002, 106,13122 .

25. Vieira, R.; Pham-Huu, C.; Keller, N.; Ledoux, M. J.; Quim. Nova 2003, 26, 665 .

26. Dresselhaus, M. S.; Dresselhaus, G.; Surihara, K.; Spain, I. L.; Goldberg, H. A.; Graphite fibers and filaments, Springer: Berlin, 1988. 